\title{
Halting progressive neurodegeneration in advanced retinitis pigmentosa
}

\author{
Susanne F. Koch, ${ }^{1,2}$ Yi-Ting Tsai, ${ }^{1,2,3}$ Jimmy K. Duong, ${ }^{4}$ Wen-Hsuan Wu, ${ }^{1,2}$ Chun-Wei Hsu, ${ }^{1,2}$ Wei-Pu Wu, ${ }^{1,2}$ Luis Bonet-Ponce, ${ }^{5}$ \\ Chyuan-Sheng Lin, ${ }^{6}$ and Stephen H. Tsang ${ }^{1,2,3,6}$ \\ 'Barbara and Donald Jonas Laboratory of Stem Cells and Regenerative Medicine and Bernard and Shirlee Brown Claucoma Laboratory, Departments of Pathology and Cell Biology, Columbia University, \\ New York, New York, USA. 'Edward S. Harkness Eye Institute, New York Presbyterian Hospital, New York, New York, USA. ${ }^{3}$ Institute of Human Nutrition, College of Physicians and Surgeons, \\ Columbia University, New York, New York, USA. ${ }^{4}$ Department of Biostatistics, Mailman School of Public Health, Columbia University Medical Center, New York, New York, USA. ${ }^{5}$ Department of Physiology, \\ School of Medicine and Dentistry, Catholic University of Valencia, Valencia, Spain. ${ }^{6}$ Herbert Irving Comprehensive Cancer Center, Columbia University Medical Center, New York, New York, USA.
}

\begin{abstract}
Hereditary retinal degenerative diseases, such as retinitis pigmentosa (RP), are characterized by the progressive loss of rod photoreceptors followed by loss of cones. While retinal gene therapy clinical trials demonstrated temporary improvement in visual function, this approach has yet to achieve sustained functional and anatomical rescue after disease onset in patients. The lack of sustained benefit could be due to insufficient transduction efficiency of viral vectors ("too little") and/or because the disease is too advanced ("too late") at the time therapy is initiated. Here, we tested the latter hypothesis and developed a mouse RP model that permits restoration of the mutant gene in all diseased photoreceptor cells, thereby ensuring sufficient transduction efficiency. We then treated mice at early, mid, or late disease stages. At all 3 time points, degeneration was halted and function was rescued for at least 1 year. Not only do our results demonstrate that gene therapy effectively preserves function after the onset of degeneration, our study also demonstrates that there is a broad therapeutic time window. Moreover, these results suggest that RP patients are treatable, despite most being diagnosed after substantial photoreceptor loss, and that gene therapy research must focus on improving transduction efficiency to maximize clinical impact.
\end{abstract}

\section{Introduction}

Retinitis pigmentosa (RP), a progressive neurodegenerative disease, is the most common cause of hereditary blindness in developed countries $(1,2)$. In the early stage of the disease, peripheral rod photoreceptor degeneration is underway, and patients experience night blindness; in addition, some cones exhibit structural pathologies (3). Rod degeneration continues in mid and late stages, causing tunnel vision. In the late stage, macular cone loss becomes significant enough that patients lose daylight vision and are often deemed legally blind (1).

There is currently no treatment for RP (indeed, for any retinal degenerative disease) that can halt the loss of both photoreceptors and function. Of those therapies on the horizon, gene therapy may be the most promising (4-6). Therapeutic development is negatively impacted by the fact that when the efficacy of a potential gene therapy is low, it is difficult to control for the various possible reasons. Failure might result if the means of delivering the therapeutic to its target cells is insufficient, or if a therapeutic is administered too late to halt the degeneration cascade. In the current study, we address these 2 potential limitations by first optimizing the means of delivery, and then by testing a gene therapeutic delivered under optimized conditions at multiple disease stages.

\section{Related Commentary: p. 3424}

Conflict of interest: The authors have declared that no conflict of interest exists. Submitted: April 22, 2015; Accepted: July 13, 2015.

Reference information: J Clin Invest. 2015;125(9):3704-3713. doi:10.1172/JCI82462.
Currently, therapeutic genes are administered by subretinal injection. However, there are 3 significant problems with this approach. First, the therapeutic generally reaches only a fraction of the diseased cells. Second, injections are inherently highly variable, leading to large deviations in response and dictating the use of large numbers of animals to compensate for that deviation. Third, injections are invasive and can lead to side effects (e.g., trauma). Thus, a method that does not involve subretinal injection would greatly facilitate the study of disease mechanisms and the development of therapeutics.

A second major issue that impacts the development and testing of any type of therapeutic is the timing of treatment. In preclinical studies, gene therapeutics are almost always delivered before the onset of cell degeneration. However, most, if not all, neural degenerative diseases are diagnosed after the onset of degeneration (7). Therefore, a therapeutic must be able to demonstrate efficacy after disease onset - even at mid- or late-stage disease. However, it has been suggested that treatment at later stages might be hindered by a "point of no return," beyond which photoreceptor cell death is unpreventable (8). An important research priority, therefore, is to determine the therapeutic time window in order to understand whether mid- or late-stage treatment is even feasible.

In the current study, we generated an RP mouse model in which mutant rod-specific cyclic GMP (cGMP) phosphodiesterase 6b (PDE6b) (Online Mendelian Inheritance in Man [OMIM] $\# 180072$ ) can be restored to WT levels in all rod photoreceptors. Mutations in the gene encoding PDE6B are responsible for 36,000 new cases of RP worldwide each year (1, 9-11). Using a tool 
A

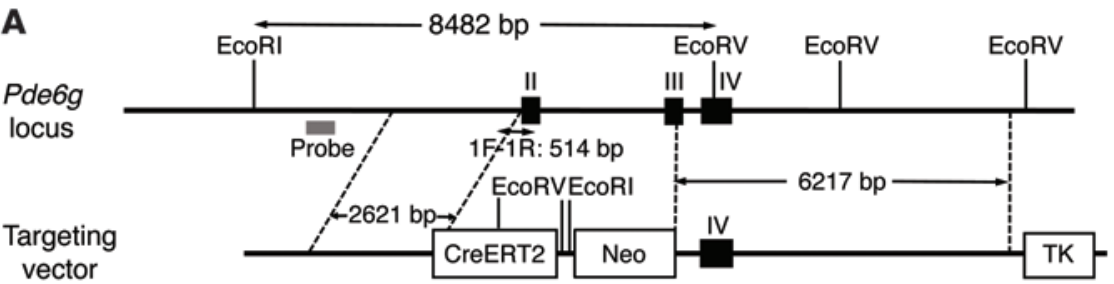

vector

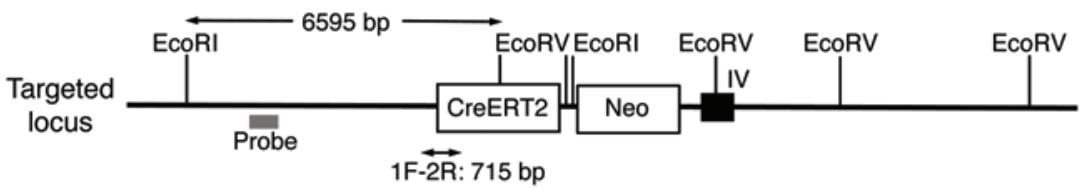

B

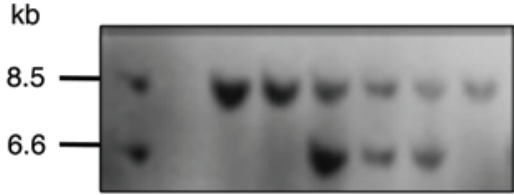

C

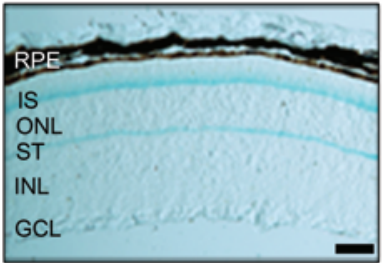

$\mathbf{E}$

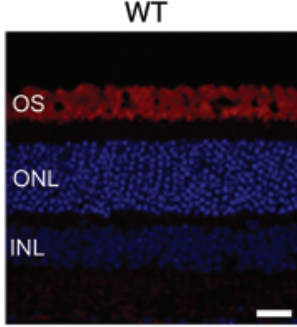

D

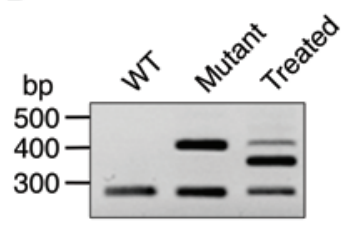

Treated
Figure 1. Inducible rod-specific Pde6 $\boldsymbol{g}^{\text {CreERT2 }}$ driver line. (A) Targeting strategy for the generation of Pde6g ${ }^{\text {CreERT2 }}$ mice. Exons 2-4 are represented by black boxes. Exons 2 and 3 were replaced by CreERT2. The targeting vector contains a neo/tk cassette (Neo). After homologous recombination, CreERT2 is placed under Pde6g locus control. (B) Southern blot analysis of EcoRI- and EcoRV-digested genomic DNA from 8 ES clones hybridized to the probe shown in $\mathbf{A}$. The 8.5- and 6.6-kb bands represent WT and targeted Pde6g alleles, respectively. (C) X-gal-stained retinal section from a 4-week-old ROSA26-lacZ Pde6g CreERT2 mouse injected with tamoxifen at 2 weeks of age. Blue, lacZ reporter gene signal. Scale bar: $50 \mu \mathrm{m}$. RPE, retinal pigment epithelium. (D) PCR analysis of ONL DNA isolated from 4-week-old retinae identified a 284-bp fragment for WT, 284- and 415-bp fragments for an untreated mutant, and 284-, 415-, and 362-bp fragments for a mutant treated with tamoxifen at 2 weeks of age. (E) Retinal sections from 4-week-old mice immunostained for PDE6b; the treated mutant was tamoxifen injected at 2 weeks of age. Scale bar: $30 \mu \mathrm{m}$. to achieve optimal gene delivery, we then systematically mapped the treatment time window for our RP gene therapeutic relative to disease progression. We demonstrate stable, sustained rescue of photoreceptor structure and visual function, even when the therapy was administered at mid-to-late disease stages.

\section{Results}

In the first part of the Results, we describe an RP mouse that allows the delivery of a therapeutic gene to all rod photoreceptors and to do so without subretinal injections. In the second part, we use this mouse model to determine whether RP can be treated well after the onset of photoreceptor cell loss.

An inducible rod-specific Cre driver. To generate a preclinical model for RP, in which the therapeutic gene can be delivered uniformly to all rods, we first generated a Pde $6 g^{\text {CreERT2 }}$ mouse and then crossed it with our Pde6b $b^{\text {Stop }} P d e 6 b^{H 620 Q}$ mice to derive Pde6 $b^{\text {Stop }}$ Pde6 $b^{\text {H620Q }}$ Pde6g GreERT2 mice (referred to herein as mutant). In the $P d e 6 b^{\text {Stop }} P d e 6 b^{H 62 Q O}$ mice, 1 allele carries a point mutation $\left(P d e 6 b^{H 620 Q}\right)$ that dramatically reduces expression of PDE6b (12, 13). This hypomorphic rod-specific mutation is not as severe as a null mutation (e.g., $P d e 6 b^{r d 1}$ ) (14) and, therefore, is a better model of human RP, which is usually caused by a missense mutation. The second allele carries a floxed stop cassette $\left(P d e 6 b^{\text {Stop }}\right)$ that prevents gene expression in the absence of Cre recombinase activity. In addition to a stop codon, the stop cassette contains 3 polyadenylation signals (3).

To generate the Pde $6 g^{\text {CreERT2 }}$ mouse, we inserted tamoxifeninducible CreERT2 into the rod-specific Pde6g locus (Figure 1A). Insertion of CreERT2 was confirmed at the genomic DNA level by Southern blot analysis (Figure 1B). To confirm the cell specificity of the Pde $6 g^{\text {CreERT2 }}$ recombinase, we crossed these mice with the ROSA26-lacZ reporter mouse line. ROSA26-lacZ reporter mice contain a loxP-flanked stop cassette preventing expression of the downstream lacZ gene; when crossed with a Cre Tg strain, the stop sequence is removed, and lacZ is expressed in cells where Cre is expressed (15). After i.p. injection of tamoxifen, the ROSA26-lacZ Pde6g ${ }^{\text {CreERT2 }}$ double-Tg mice showed X-gal staining in photoreceptor cells, but not in cells in the inner nuclear layer (INL) or ganglion cell layer (GCL) (Figure 1C). The subcellular pattern of X-gal staining in photoreceptors - in the inner segment (IS) and synaptic terminals (STs) - has been previously described (16). These data validate functional Cre recombinase expression in photoreceptors.

To demonstrate that $P d e 6 g^{\text {CreERT2 }}$ can remove the stop cassette in the Pde6 $b^{\text {Stop }} P d e 6 b^{\mathrm{H} 62 \mathrm{QO}}$ mice, we treated mutant mice with tamoxifen and then performed PCR analysis on DNA isolated from the retinal outer nuclear layer (ONL). A 362-bp band was found in DNA amplified from treated mutant mice, but not in DNA 


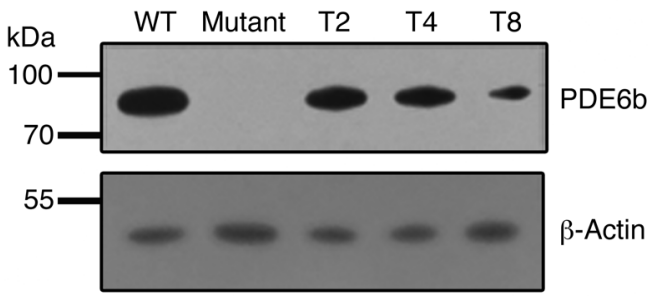

amplified from the ONL of untreated mutant or WT retinae (Figure 1D). A faint 415-bp nonrecombined product was also noted; this is because, in addition to rods, the ONL also contains cone nuclei, in which Pde6 $g^{\text {CreERT2 }}$ recombinase is inactive. To analyze expression of PDE6 b protein after tamoxifen-induced Pde $6 g^{\text {CreERT2 }}$ mediated recombination, retinal sections were immunolabeled with an Ab against PDE6b. While immunolabeling was absent from mutant retinae, the outer segment (OS) of treated and WT retinae exhibited intense levels of staining (Figure 1E). These data confirm the efficient, near-complete tissue-specific Pde6g CreERT2 mediated recombination.

These reporter gene and target gene experiments demonstrate the high efficiency of our inducible rod-specific Cre driver. With this tool, we can restore PDE6b expression in all mutant rods to levels that are close to those of WT, and we can do so at specific time points. In the following experiments, in addition to our mutant mice, we used $P d e 6 b^{+} P d e 6 b^{H 620 Q}, P d e 6 g^{C r e E R T 2}$ mice as a WT (positive) control.

Photoreceptor degeneration in Pde6 $b^{\text {Stop }} P d e 6 b^{H 620 Q}, P d e 6 g^{\text {CreERT2 }}$ mutant mice. To test our gene therapy after the onset of photoreceptor degeneration at early, mid, and late disease stages, we first needed to gain a basic understanding of the disease progression timeline in our mutant. To do this, we performed a quantitative histological analysis of the mutant and WT photoreceptor layer, known as the ONL, from mice that were 2,4 , or 8 weeks of age. We measured ONL thickness, cone numbers, and cone and rod photoreceptor OS lengths.

At 2 weeks of age, OS and ONL morphology in the mutant retinae (Supplemental Figure 1, A and D; supplemental material available online with this article; doi:10.1172/JCI82462DS1) were indistinguishable from that of age-matched WT control retinae (not shown). It is important to note that in 2-week-old retinae, developing retinal OS maturation and programmed photoreceptor cell death are ongoing $(17,18)$; as a result, the 2-week-old mutant rod OS was reduced, and the ONL was thicker than in WT adult retinae (Supplemental Figure 1, G and H). Cone OS lengths and cone numbers were not quantified at 2 weeks, since expression of the cone marker was low (Supplemental Figure 1D). At 4 weeks of age, rod OS length and ONL thickness were reduced by roughly $50 \%$ and $30 \%$, respectively $(P<0.015$ and $P<0.036)$ compared with WT adult retinae (Supplemental Figure 1, B and G). In contrast, cone OS length and cone numbers were not significantly changed ( $P=0.65$ and $P=0.37$, respectively) (Supplemental Figure $1, \mathrm{E}, \mathrm{I}$, and J). At 8 weeks of age, OS length and ONL thickness were dramatically deceased - by about $70 \%$ - compared with WT adult retinae $(P<0.009$ and $P<0.001$, respectively) (Supplemental Figure 1, C, G, and $\mathrm{H}$ ). In addition, cone OS length and cone numbers were dramatically decreased - by roughly $65 \%$ and
Figure 2. Restoration of PDE6b expression at early, mid, and late stages of RP. Two-, four-, and eight-week-old mutant mice were treated with tamoxifen (T2, T4, and T8, respectively). When mice were 20 weeks old, retinae were lysed, and Western blotting was performed for PDE6b. Blots for WT and untreated mutant retinae are also shown. Immunoblotting was performed in triplicate. A representative immunoblot is shown. $\beta$-actin was used as a loading control.

30\%, respectively $(P<0.003$ and $P<0.023)$ (Supplemental Figure $1, \mathrm{~F}, \mathrm{I}$, and J). Given this progressive decrease in ONL thickness and OS length, we defined the 2-, 4-, and 8-week-old mutant retinae as being in early, mid, and late stages of RP disease.

Restoration of PDE6b expression and retinal function over a broad time window. To determine the therapeutic time window for photoreceptor rescue, we injected tamoxifen into 2-, 4-, and 8-week-old mice (i.e., at early, mid, and late disease stages, respectively). To determine whether PDE6b expression was rescued by the treatment, retinae were subjected to immunoblot analysis (Figure 2). PDE6b expression (i.e., a 90-kDa band) was observed in mutant mice treated at all 3 stages, but the level of expression was highest when tamoxifen was administered at 2 weeks of age. This progressive decrease in expression of rod-specific PDE6b correlates temporally with the decrease noted in rod OS length and ONL thickness (Supplemental Figure 1, A-C).

To assess the degree to which retinal function was rescued, we performed electroretinographic (ERG) analysis in 18-weekold mice. Under dark-adapted conditions with dim flashes, to derive rod responses, WT retinae exhibited a rod-specific b-wave (positive deflection), and mutant retinae showed no response; tamoxifen treatment rescued the rod-specific b-wave, with earlier treatment eliciting a higher response (Figure 3A). Under darkadapted conditions with bright flashes, to derive mixed rod and cone responses, WT retinae exhibited an a-wave (negative deflection), followed by a b-wave. Mutant retinae lacked the a-wave and exhibited a strongly reduced $b$-wave; tamoxifen treatment led to nearly complete restoration of the a- and b-waves at the 2- and 4 -week treatment time points and partial restoration at the 8-week time point (Figure 3A). Under light-adapted conditions with very bright flashes, to derive cone responses, WT retinae exhibited a cone-specific b-wave, which was strongly reduced in mutant mice; treatment at every stage rescued the cone responses (Figure 3A).

We quantified the amplitudes of the $\mathrm{a}$ - and $\mathrm{b}$-waves, comparing treated and untreated mutants (Figure 3, B-E). Treatment at 2 and 4 weeks (i.e., early- and mid-stage disease, respectively) significantly increased the rod b-wave amplitude $(P<0.001)$. Treatment at 8 weeks (late-stage disease) appeared to rescue rod ERG responses, however, the effect was not statistically significant $(P=0.070)$ (Figure $3 \mathrm{~B})$. This is probably because approximately $70 \%$ of rods had died by 8 weeks (Supplemental Figure 1 ). The b-wave amplitudes in light-adapted ERG responses were significantly greater in treated mice at 2,4 , and 8 weeks of age $(P<0.003$, $P<0.001$, and $P<0.011$, respectively); this indicates that treatment prevented the progressive degeneration of cones (Figure $3 C)$. Treated mice also exhibited significantly greater mixed rodcone a-wave amplitudes at all 3 treatment time points (2 and 4 weeks, $P<0.001$; 8 weeks, $P<0.049$ ) (Figure 3D). Similarly, the 


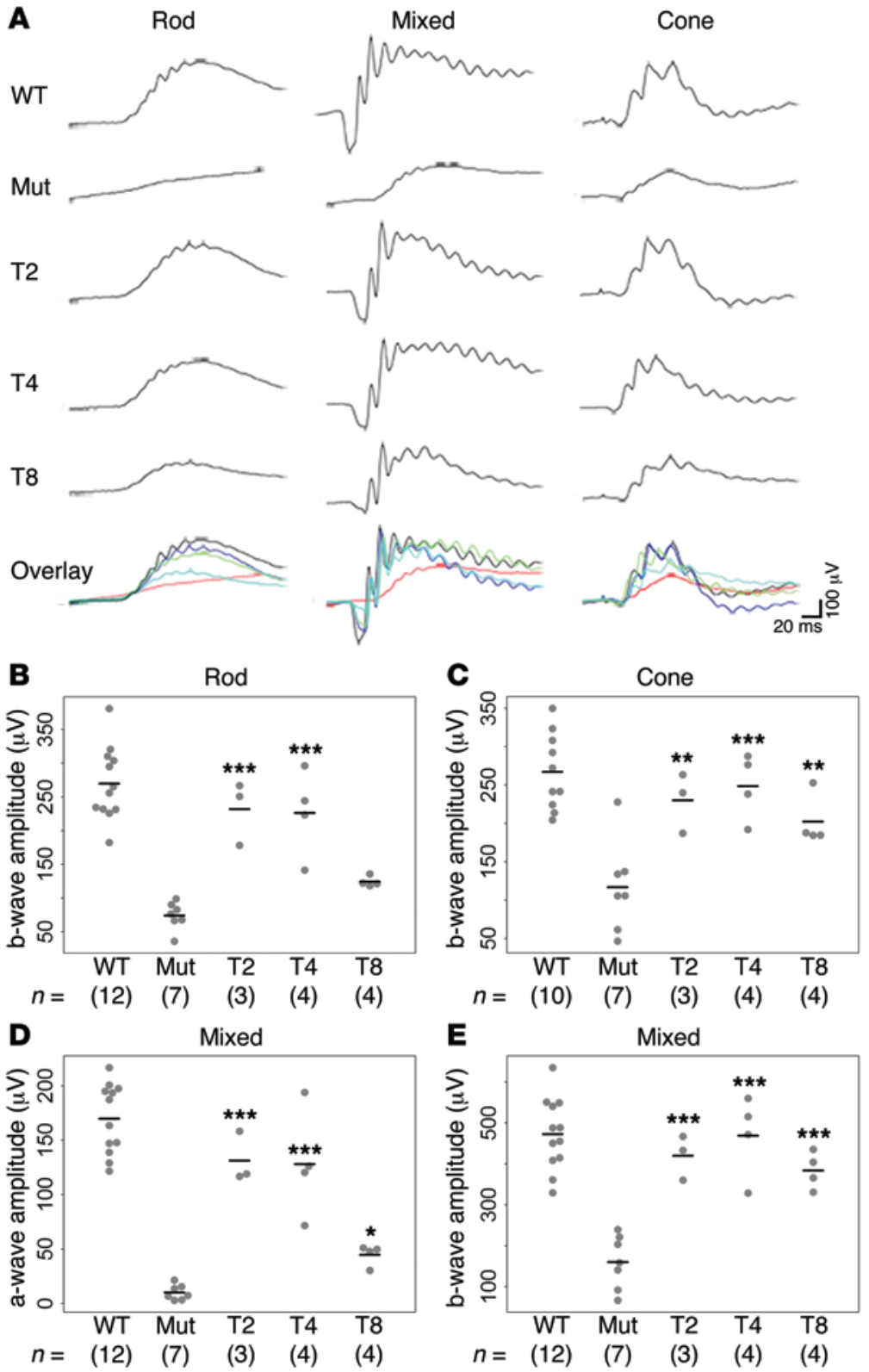

Figure 3. Preservation of retinal function after gene therapy administered at early, mid, and late disease stages. WT, mutant, and treated mice were measured at week 18. (A) Representative ERG responses for rods, mixed rods and cones, and cones from WT (black, WT), mutant (red, Mut), and treated mice. The treated mice were tamoxifen injected at 2 weeks (dark blue, T2), 4 weeks (green, T4), and 8 weeks (light blue, T8) of age. (B-E) Statistical analysis of ERG amplitudes for the scotopic rod-specific b-wave (B), the photopic cone-specific b-wave (C), the mixed rod-cone-specific a-wave (D), and the mixed rod-cone-specific b-wave (E). Gray dots represent individual mice (for each mouse, the 2 eyes were averaged; $n$ values are shown below each group), and horizontal lines represent the group means. A linear regression model was fit to compare groups. ${ }^{*} P<0.05,{ }^{* *} P<0.01$, and ${ }^{* *} P<0.001$ for significant differences between treated and untreated mutant groups using linear regression model.

ease progression manifests structurally as shortening of the rhodopsin-positive rod OS, followed by loss of the rod itself (19). In fact, in WT retinae, we observed intense rhodopsin staining in the OS (Figure 4A) that was almost completely absent in mutant retinae (Figure $4 \mathrm{~B}$ ). In retinae of mice treated at the early 2-week time point, the OS length was comparable to that of WT retinae (Figure 4C). OS length was also rescued at 4 and 8 weeks (Figure 4 , D and E); that is, the gene therapy prevented any further decrease in OS length.

This rod cell loss manifests structurally as decreased thickness of the ONL. We therefore stained sections of 20-week-old retinae with Hoechst dye, which labels all nuclei. Retinae treated at 2 and 4 weeks had thicker ONLs compared with those of untreated mutants (Figure 4). Retinae treated at 8 weeks had ONLs that were also thicker than those of untreated retinae, but the difference at this late stage was smaller, because the majority of rods had died by 8 weeks (Supplemental Figure 1). In RP, rod degeneration leads to the secondary degeneration of cones. To specifically examine the response of cones to our treatment, retinal sections were immunostained for cone arrestin. In WT retinae, immunostaining was apparent in cone pedicles, axons, mixed rod-cone $\mathrm{b}$-wave amplitudes were significantly increased at all 3 treatment time points $(P<0.001)$ (Figure $3 \mathrm{E}$ ).

Thus, we were able to demonstrate functional rescue of photoreceptors, even at late disease stages. On the other hand, earlier treatment was associated with stronger ERG responses. Mutant mice treated at weeks 2 or 4 exhibited responses (rod, mixed, and cone) that were similar to those of WT mice. Treatment at week 8 led to more modest increases in ERG amplitudes, especially rod responses. Since ERG amplitudes depend on photoreceptor numbers, our data suggest that treatment directly prevented further rod degeneration and indirectly prevented cone degeneration as well.

Halt of photoreceptor degeneration over a broad time window. We next tested whether treatment at different stages of disease progression was able to rescue photoreceptor cells. To do this, we measured OS length in sections of retina from 20-week-old mice (WT, mutant, and treated mutant) that were immunostained for rhodopsin, which is highly expressed in normal rod OSs. RP dis- and in the cytoplasm of cone ISs and OSs (Figure 4F). In mutant retinae, cone arrestin immunostaining suggested that both the number of cones and the length of their OSs were reduced (Figure $4 \mathrm{G})$. Tamoxifen injection at 2, 4 , or 8 weeks of age preserved cone numbers and structure (Figure $4, \mathrm{H}-\mathrm{J}$ ).

We next quantified the observed changes in OS length and ONL thickness. Rod OS length and ONL thickness were significantly greater in retinae of mice treated at either 2 or 4 weeks compared with retinae of untreated mutants $(P<0.001)$; on the other hand, there was no significant difference between mice treated at 8 weeks and untreated mutants $(P=0.7$ and $P=0.2$, respectively) (Figure $4, \mathrm{~K}$ and $\mathrm{L}$ ). On the other hand, cone OS length was significantly greater in mice treated at 2 or 4 weeks $(P<0.001)$, or at 8 weeks $(P<0.05)($ Figure $4 \mathrm{M})$. Thus, while we were able to rescue the remaining photoreceptors even at late-stage disease, earlier treatment (when there are more photoreceptors) was associated with greater functional and structural rescue (Figures 3 and 4). 

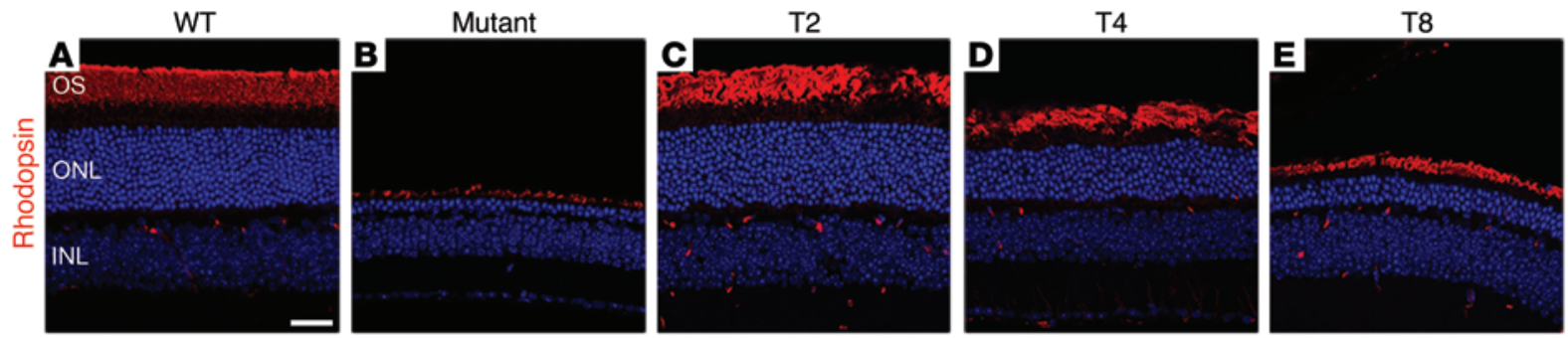

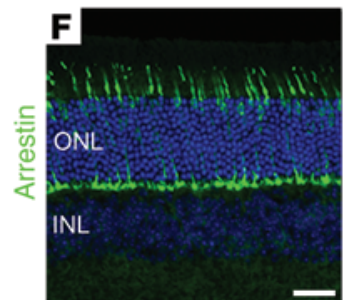

$\mathbf{K}$

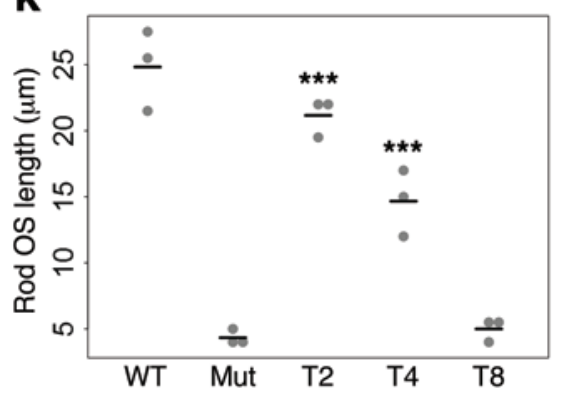

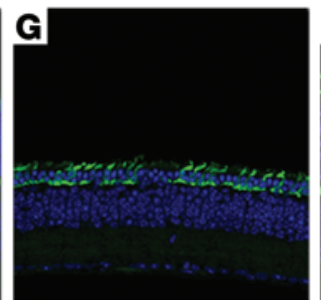

$\mathbf{L}$

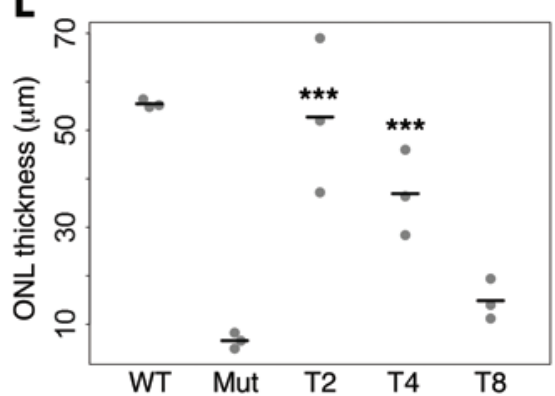

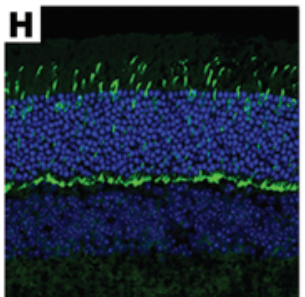

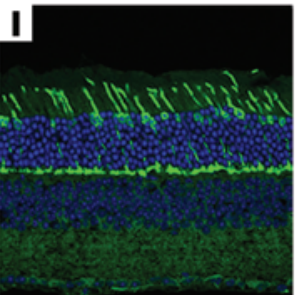

M
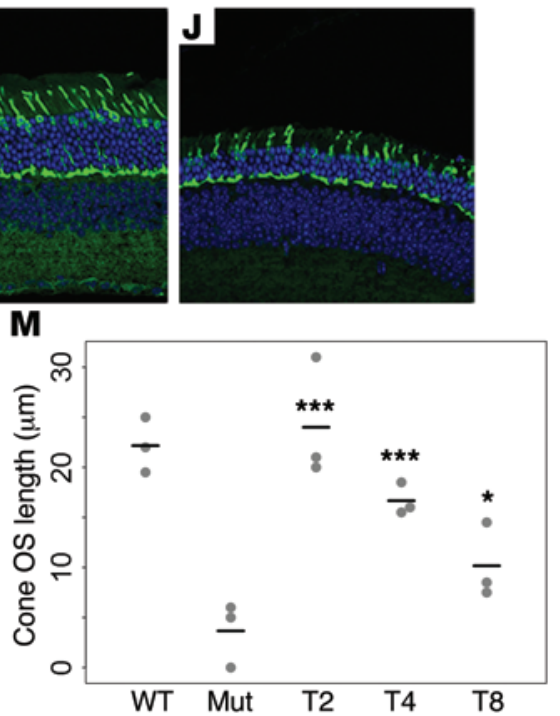

Figure 4. Preservation of retinal structure after gene therapy administered at early, mid, and late disease stages. WT, mutant, and treated retinae were sectioned and immunostained at week 20. Treated mice were tamoxifen injected at 2 weeks (T2), 4 weeks (T4), and 8 weeks (T8) of age. (A-E) Images show anti-rhodopsin Ab (red) labeling of rod OSs. (F-J) Images show anti-cone arrestin Ab (green) labeling of cones. Nuclei were stained with Hoechst dye (blue). (K-M) Quantification of rod OS length (K), ONL thickness (L), and cone OS length (M). Each gray dot represents an individual mouse ( $n=3$ for each group). Horizontal lines represent the group means. A linear regression model was fit to compare groups. ${ }^{*} P<0.05$ and ${ }^{* *} P<0.001$ for significant differences between treated and untreated mutant groups using linear regression model. Scale bars: $30 \mu \mathrm{m}$.

Absence of reactive gliosis after gene therapy. Reactive gliosis is an indicator of damage to the CNS, including damage caused by significant levels of cell degeneration. In retina, gliosis is indicated by upregulation of glial fibrillary acidic protein (GFAP) in Müller glial cells. To determine whether our gene therapy reduces retinal damage, we performed GFAP immunolabeling on sections of 20-week-old retinae. In WT retina, GFAP immunolabeling was apparent in the endfeet of Müller glia (Figure 5A). In untreated mutant retina, GFAP immunolabeling was increased, with intensely labeled stress fibers extending to the ONL (Figure 5B); this activation of Müller glia was apparent in 4-week-old untreated mutant retinae (but not in 2-week-old retinae) and was even more severe by 8 weeks (Supplemental Figure 2 ). In retinae of mutant mice treated at 2,4 , and 8 weeks of age, the GFAP immunolabeling pattern was comparable to that seen in WT retinae (Figure 5, C-E). Thus, treatment prevented Müller glial cell activation and infiltration at an early disease stage and reversed it at mid and late stages.

Long-term effects of treatment. Figures 2-5 demonstrate functional and structural rescue of diseased rods in our PDE6b-deficient RP mouse model. In addition to this direct rescue by gene therapy, data in Figures 3 and 4 provide evidence for indirect, sec-
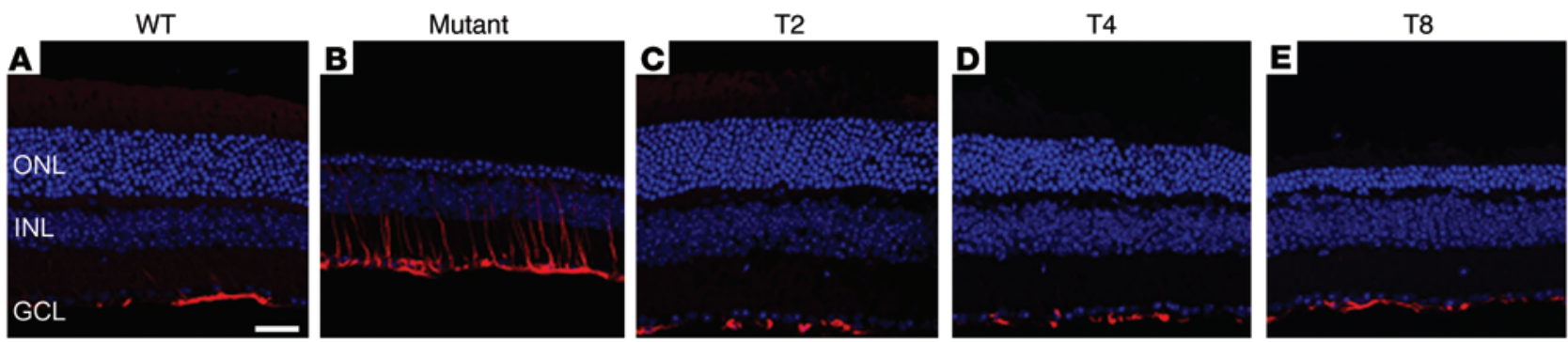

Figure 5. Absence of gliosis in treated mutant retina. WT, mutant, and treated retinae were sectioned and immunostained at week 20 . The treated mice were tamoxifen injected at 2 weeks (T2), 4 weeks (T4), and 8 weeks (T8) of age. (A-E) Anti-GFAP Ab (red) was used to label reactive phenotype in Müller glia; nuclei were stained with Hoechst dye (blue). IHC was performed on 3 mice for every group. A representative immunostaining is shown. Scale bar: $30 \mu \mathrm{m}$. 

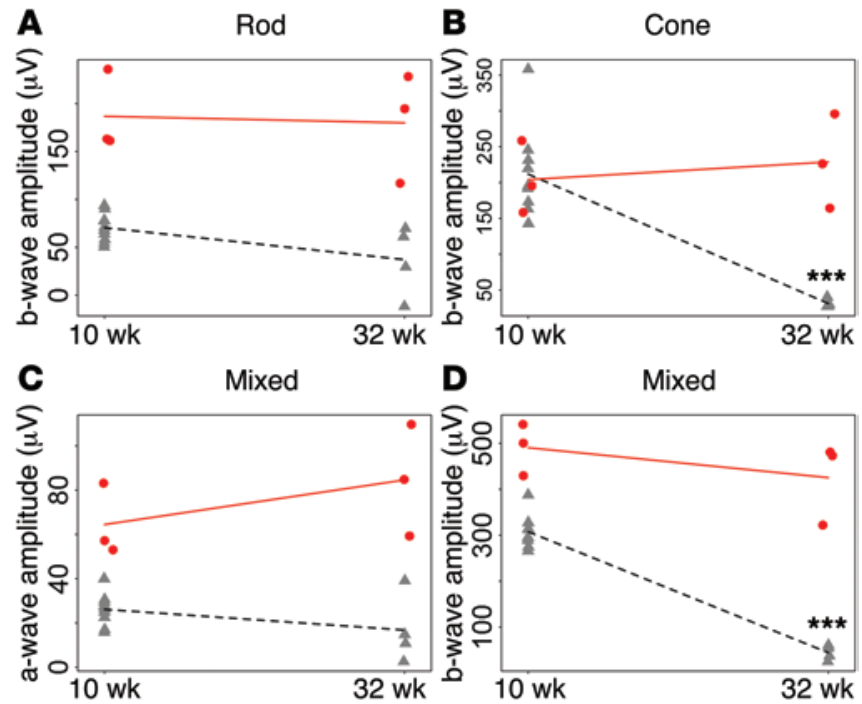

Figure 6. Stable rescue of visual function following treatment at a late disease stage. At 8 weeks of age, mutant mice were tamoxifen injected or not; at 10 and 32 weeks of age, ERG responses were recorded. (A)Rodspecific b-wave, (B) photopic cone-specific b-wave, (C) mixed rod-conespecific a-wave, and (D) mixed rod-cone-specific b-wave. Red dots represent individual treated mutant mice ( $n=3$ for each time point). Gray triangles represent individual untreated mutants $(n=10$ and 4 at 10 and 32 weeks of age, respectively). Red solid lines and black dashed lines connect the group means of the treated and untreated mutant mice, respectively. For each mouse, the 2 eyes were averaged. A paired $t$ test was used to compare weeks 10 and 32 for the treated group. A 2-sample $t$ test was used to compare weeks 10 and 32 for the untreated mutant group. ${ }^{* * *} P<0.001$ for significant differences between weeks 10 and 32 . ondary rescue of cones. To analyze whether these rescue effects are stable, and thus long lasting, we treated mice at 8 weeks of age and analyzed retinal function at 10 and 32 weeks of age and retinal structure at 10, 20, and 32 weeks of age.

In retinae of treated mutant mice, ERG responses at 10 and 32 weeks of age were not significantly different $(P=0.8, P=0.6$, and $P=0.1$ for rod, cone, and mixed responses, respectively) (Figure 6). In contrast, in untreated mutant mice, cone and mixed b-wave amplitudes decreased significantly between 10 and 32 weeks $(P<0.001)$. Rod $b$-wave and mixed a-wave amplitudes (i.e., rod-based ERG measures) were quite low, and the modest decrease between 10 and 32 weeks was not significant $(P=0.2$ and $P=0.3$, respectively) (Figure 6 ). We also examined treatment stability by immunolabeling rod OSs with rhodopsin Ab (Figure $7, \mathrm{~A}-\mathrm{F}$ ) and cones with cone arrestin $\mathrm{Ab}$ (Figure 7, G-L); a nuclear stain was used to label the ONL. Over the 22-week study, mutant retinae exhibited rod os shortening, progressive and dramatic loss of cones, and thinning of the ONL. In treated retinae, on the other hand, these structures did not appear to change. These experiments demonstrate that the gene therapy at a relatively late disease stage prevented degeneration of the remaining rods and cones for up to 6 months.

We have quantitated ONL thickness in WT and untreated mutant mice at 2, 4, and 8 weeks of age (Supplemental Figure 1 ), as well as in WT, untreated, and treated mutant mice at 20 weeks of age (Figure $4 \mathrm{~L}$ ). We next examined ONL thickness in WT, untreated mutant, and treated mutant mice at 1 year and then combined all of these data (Figure 8). In WT mice (Figure 8, blue line), the decrease in ONL thickness was due to
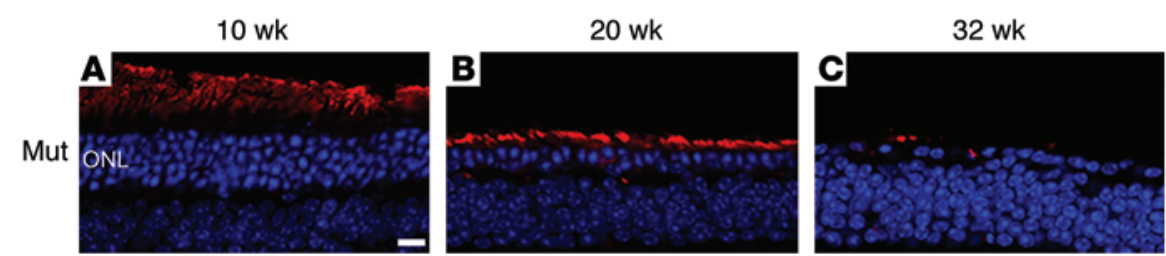

T8
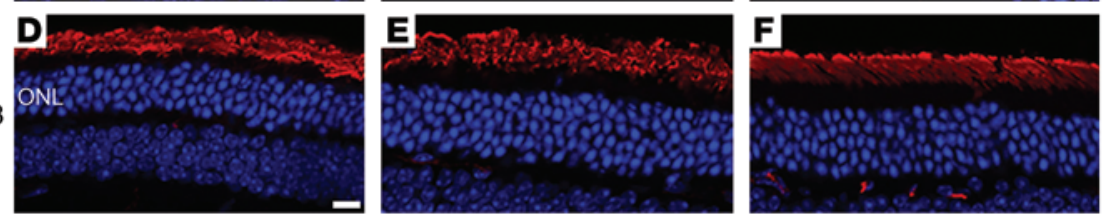

Mut
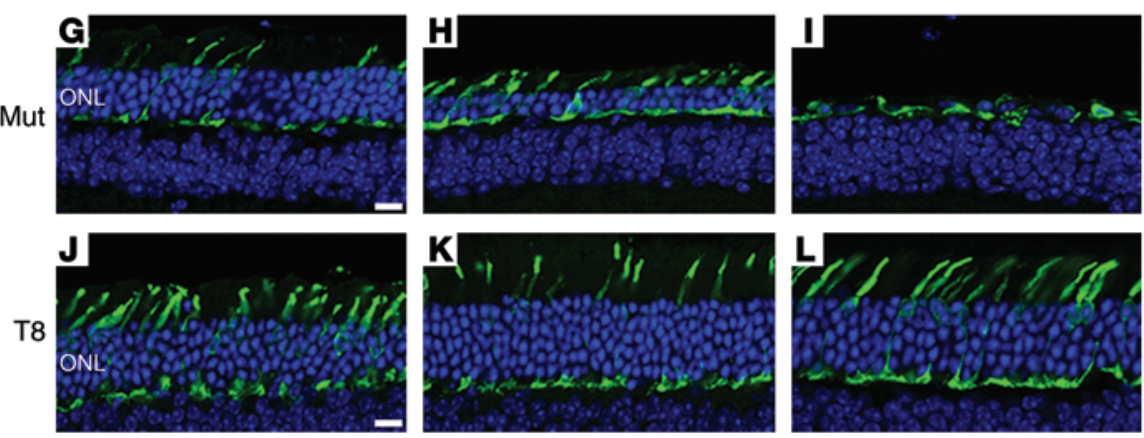

Figure 7. Stable rescue of retinal structure following treatment at a late disease stage. At 8 weeks of age, mutant mice were tamoxifen injected (T8) or not (Mut). Mice were then sacrificed at 10, 20, or 32 weeks of age, and retinae were sectioned and immunostained; nuclei were stained with Hoechst dye (blue). (A-F) Images show anti-rhodopsin Ab (red) labeling of rod OSs. (G-L) Images show anti-cone arrestin Ab (green) labeling of cones. Scale bars: $10 \mu \mathrm{m}$. 


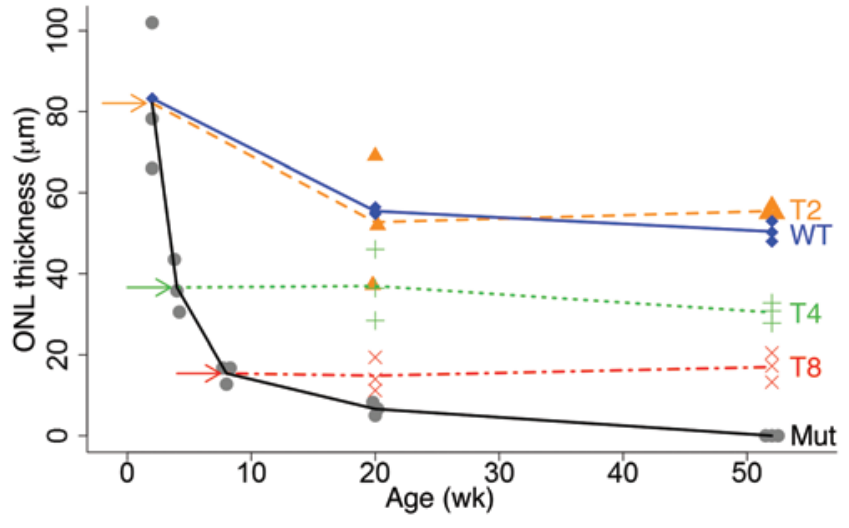

Figure 8. Photoreceptor degeneration is halted at all treatment time points. Quantification of ONL thickness from WT (blue), mutant (black), and treated mice. Treated mice were tamoxifen injected at 2 weeks (yellow, T2), 4 weeks (green, T4), and 8 weeks (red, T8) of age. Arrows indicate the treatment time points. Each symbol represents an individual mouse $(n=3$ for each group at 52 weeks). Data for the untreated mutant at weeks 2, 4, and 8 are taken from Supplemental Figure 1B; data for week 20 are taken from Figure $4 \mathrm{~L}$.

\section{Discussion}

In the present study, we generated and validated a tamoxifen-inducible Cre recombinase driver mouse (Pde6g CreERT2) (Figure 1, $\mathrm{A}-\mathrm{C}$ ) that can be crossed with a floxed mouse to conditionally activate a gene (as we have done in this study) or delete any gene exclusively in rods. This tool allowed us to uniformly deliver a gene therapeutic to all rods in the retina, while also eliminating the problems associated with subretinal injections. We believe that this tool will have a substantial impact, because it provides a highly reproducible and precise method for generating and studying "optimally treated" retinae. It will be especially helpful when the gene of interest is ubiquitously expressed (e.g., genes causing embryonic or neonatal lethality).

In the current study, we crossed our Pde6g $g^{\text {CreERT2 }}$ driver with our Pde6b $b^{\text {stop }}$ Pde $6 b^{H 620 Q}$ mice (3) to derive our mutant Pde6 $b^{\text {Stop }}$ $P d e 6 b^{H 620 Q}, P d e 6 g^{\text {CreERT2 }}$ mouse. With this mutant, we were able to conditionally activate expression of $P d e 6 b$ in a rod-specific manner, over the entire retina. By administering tamoxifen at early, mid, and late disease stages, we mimicked the clinical scenario in which patients are typically diagnosed after onset of degeneration, with the majority already exhibiting significant loss of rods and cones in their peripheral retinae (7). In contrast, most previous animal studies administered the gene therapy before the onset of degeneration $(20,21)$. Our data clearly show significant and sustained functional and structural rescue at all treatment time points. These data represent 2 findings: first, that rod gene therapy can stop both rod and cone degeneration and second, that it can do so even at mid and late disease stages. The data also demonstrate a broad time window for gene therapy in our preclinical RP model (Figure 8). These findings are consistent with the "one-hit" model of cell death, in which mutation imposes a mutant steady state on the neuron, and a single event randomly initiates cell death. According to this theory, rescue is not diminished by age, although fewer neurons are available to rescue as the disease progresses (22).
In recent retinal gene therapy clinical trials, some of the research subjects showed functional improvement (23-30). However, several follow-up studies clearly showed that photoreceptor loss had not been halted or even slowed (31-34). It was suggested that there is a threshold of accumulated changes as a result of the mutation, after which photoreceptor death is inevitable (31); this has been called the "point of no return" (8). If such a threshold (i.e., point of no return) exists, our mouse data demonstrate that it would have to occur at very terminal disease stages - after our late stage (i.e., 8 weeks of age). Instead, our data suggest that the failure to rescue photoreceptors in humans, rather than being the result of a point of no return, is due to transduction efficiencies that are therapeutically insufficient. Insufficient transduction would allow abnormalities in untreated mutant cells to continue to stress both treated and untreated photoreceptors. This same logic could be used to explain the failure to halt degeneration in some previous animal studies $(13,35)$. In support of this idea are gene therapy studies demonstrating a delay in photoreceptor degeneration that was limited to the transduced area (36-38).

Although RP is typically diagnosed in young adulthood, photoreceptor degeneration has been detected in RP patients as young as 6 years of age (1). Therefore, for RP gene therapy, it is especially important to be able to demonstrate long-term efficacy that is, stability that will last over the many remaining decades of a patient's life. In addition, any therapy that establishes longterm rescue of cones could have enormous clinical impact, since humans spend most of their waking hours in light levels that are within the dynamic range of cones (i.e., cone loss is blinding). In the current study, we were able to demonstrate long-term stability of both functional and structural rescue (Figures 6-8). In fact, ours is the first study, to our knowledge, to demonstrate a stable long-term halt of rod and cone degeneration. There are probably multiple mechanisms underlying this non-cell-autonomous rescue of cones. First, our direct rescue of rods reduced the level of cell death in the RP retinae, which may lead to an increase in positive trophic factors (39) and a decrease in toxic factors. For example, we demonstrated that gene therapy reverses Müller glial cell activation (Figure 5 and Supplemental Figure 2), which, by itself, is likely to exert a positive impact on cell survival (40).

Our findings in Pde6b-mutant mice are likely to be generalizable to humans, because our RP mouse model recapitulates much of human RP disease progression. Specifically, disease progression in both our mutant and RP patients is characterized by early loss of rods, followed by secondary loss of cones (1). In both our mutant and human RP patients, the structural and functional phenotypes manifest as changes in ONL thickness and ERG recordings (41). To develop an effective RP gene therapy for humans, our work points to the importance of improving delivery of gene therapeutics and delivering those therapeutics at the earliest point possible. To improve vector delivery (i.e., to increase both the area treated as well as the percentage of target cells treated), it is important to explore newer adeno-associated virus (AAV) vector variants and promoters, generated to enhance transduction efficiency and specificity $(42,43)$, and to test single versus repeated administration of these AAVs. In addition, a new AAV variant has been evolved to efficiently target cells in the retina from an intravitreal injection (44). 
Our work strongly suggests that RP is treatable with gene therapy, even at late disease stages. On the other hand, at very advanced stages - when few or no photoreceptors remain - gene therapy is not an option, and other therapies (e.g., stem cell-based transplantations, ref. 45; optogenetics, refs. 46, 47; or retinal prosthesis, ref. 48) could be considered. Further, our work suggests that to minimize cell loss and maximize visual function, it is critical to intervene with an effective gene therapy (such as ours) when photoreceptor loss is still modest. This can be accomplished with better diagnostic tools and education. The combination of improved delivery of gene therapies and earlier diagnosis promises to effectively halt the disease in its earliest stages.

\section{Methods}

Animals. ROSA26-lacZreporter mice (B6.129S4-Gt(ROSA)26Sor ${ }^{\text {tmlSor }} / \mathrm{J}$ ) were purchased from The Jackson Laboratory. Pde $6 b^{H 620 Q}$ mice were re-derived via oviduct transfer from morulae provided by the European Mouse Mutant Archive (EMMA) (12,13). Pde $6 b^{\text {Stop }}$ mice were generated in the Tsang laboratory, as previously described (3). Pde6g $g^{\text {CreERT2 }}$ mice were generated as described below. All mice were maintained in the Columbia University Pathogen-free Eye Institute Annex Animal Care Services Facility under a 12-hour light/12-hour dark cycle.

Generation of Pde6g $g^{\text {CrEERT2 }}$ driver mice. KV1 embryonic stem (ES) cells were resuspended in saline at a density of $4 \times 10^{7}$ cells $/ \mathrm{ml}$ and infected with a linearized targeting vector by electroporation (BioRad electroporator, single pulse, $220 \mathrm{~V}$ and $960 \mu \mathrm{F})(3,49,50)$. Infected cells were immediately plated on three $10-\mathrm{cm}$ dishes containing mitomycin-c-treated neomycin-resistant primary mouse embryonic fibroblasts, and the medium was replaced 24 hours later. After 1 month in culture, the ES cell DNA was screened for gene replacement using Southern blot analysis; DNA was digested with EcoRI and EcoRV and the fragments hybridized to the probe shown in Figure $1 \mathrm{~A}$ to discriminate between targeted $(8.5 \mathrm{~kb})$ and endogenous WT $(6.6 \mathrm{~kb})$ fragments (Figure 1B).

Blastocysts were harvested from pregnant $\mathrm{C} 57 \mathrm{BL} / 6 \mathrm{~J}$ mice at 3.5 days postcoitus by insertion of a syringe-attached needle into the cervical region on one side of the uterus, which was then flushed (50). Blastocysts were extracted by mouth pipetting with a drawn-out capillary tube and placed in a culture dish containing $37^{\circ} \mathrm{C} \mathrm{CO}_{2}$-equilibrated potassium simplex optimized medium (KSOM). The targeted ES cells were thawed, passaged once, washed with PBS, dissociated with trypsin, and then plated on a gelatinized plate. After 1 hour at $37^{\circ} \mathrm{C}$, the plate was rinsed, the supernatant centrifuged, and the pelleted cells resuspended in $0.1 \mathrm{ml}$ of injection medium; $10-15$ targeted ES cells were injected into each C57BL/6-Tyr $r^{-2 j} / \mathrm{J}$ blastocyst and the injected blastocysts surgically reimplanted into the uterus of a pseudopregnant mother. The contribution of (agouti) KV1 ES cells to chimerism was calculated as the percentage of agouti hair on a blackhaired C57BL/6J (blastocyst-derived) background. At 6 weeks of age, male chimeras were housed with C57BL/6J-Tyr ${ }^{c-2 j} / \mathrm{J}$ females to test for germline transmission of the ES genome. ES cell-derived progeny were identified by coat color and screened for the presence of the targeted allele on the basis of the gain of an EcoRV site. Tg determination was performed using multiplex PCR and 3 primers: 1 forward, 5'-GGTCAGATTCCAGTGTGTGGG-3'; 1 reverse, 5'-CTTAGGTGGTCCTTTCCTGGG-3'; and 2 reverse, 5'-GTTTAGCTGGCCCAAATGTTG-3'.
Tamoxifen injection and PCR recombination assay. Tamoxifen (100 mg/ml in ethanol; catalog T5648; Sigma-Aldrich) was thoroughly mixed at $42^{\circ} \mathrm{C}$, diluted with corn oil to a final concentration of $10 \mathrm{mg} / \mathrm{ml}$, and then injected at a concentration of $100 \mu \mathrm{g} / \mathrm{g} \mathrm{BW}$ on 3 consecutive days ( 1 injection/day), beginning at the time points indicated in the Results section.

To assay for recombination, ONL DNA was extracted from $10-\mu \mathrm{m}$ frozen sections using a 22-gauge needle and a surgical microscope (m690; Leica). PCR was performed as previously described (3). To target the $P d e 6 b$ DNA sequence, we used 3 primers: forward primer, 5'-TGCTCTGTGGTGTTGCTCTGC-3'; reverse primer, 5'-TGGCGATGCAGAGTGTCCTGA-3'; and internal primer, 5'-GTCCTGCACGACGCGAGCTG-3'. The PCR-amplified vector sequence yielded 3 fragments: 284 bp for H620Q/WT, 415 bp for stop, and 362 bp after recombination.

IHC and morphometry. Mice were euthanized according to established IACUC guidelines and previously described procedures (36). At that point, eyes were enucleated and placed in $4 \%$ paraformaldehyde for 5 minutes. Retinae were then dissected from the eyecup, fixed in $4 \%$ paraformaldehyde for 45 minutes, washed in $0.1 \mathrm{M}$ phosphate buffer (PB), cryoprotected in 30\% sucrose overnight, and embedded. Retinae were then sectioned vertically at $10 \mu \mathrm{m}$ using a Leica cryostat. Sections were washed with $0.1 \mathrm{M} \mathrm{PB}$ and incubated overnight at $4^{\circ} \mathrm{C}$ in primary $\mathrm{Ab}$ in $0.1 \mathrm{M} \mathrm{PB}$ containing $5 \%$ ChemiBLOCKER (catalog 2170; EMD Millipore) and 0.3\% Triton X-100. The following Abs were used: rabbit anti-cone arrestin (1:5,000; catalog AB15282; EMD Millipore); mouse anti-rhodopsin (1:500; catalog sc-57432; Santa Cruz Biotechnology Inc.); and Cy3-coupled anti-GFAP (1:800; catalog C9205; Sigma-Aldrich). Subsequently, sections were washed in 0.1 M PB, and goat anti-mouse Alexa 555-conjugated secondary Ab (1:500; catalog A-21425; Molecular Probes, Life Technologies) or goat anti-rabbit Alexa 488-conjugated secondary Ab (1:500; catalog A-11070; Molecular Probes, Life Technologies) was applied for 2 hours at room temperature. Sections were then washed with $0.1 \mathrm{M}$ $\mathrm{PB}$, incubated for 5 minutes in $5 \mu \mathrm{g} / \mathrm{ml}$ Hoechst 33342 (catalog H1399; Molecular Probes, Life Technologies), and analyzed using a confocal microscope (Nikon A1).

Quantitative analyses of ONL thickness, OS lengths, and cone numbers were performed on sections containing the optic nerve (5 sections per eye). Measurements were performed $250 \mu \mathrm{m}$ from the optic nerve using Image J software (NIH). Cones were counted in $200 \times 200 \mu \mathrm{m}$ fields.

LacZ staining. ROSA26-lacZ mice were bred with Pde6g $g^{\text {CreERT2 }}$ mice. Tamoxifen was injected into the 2-week-old offspring. Two weeks later, retinae were sectioned, stained with X-gal to detect $\beta$-gal activity (LacZ Tissue Staining Kit; InvivoGen), and analyzed using a Leica DM 5000B microscope.

Immunoblot analysis. Retinae were collected from 20-week-old mice, homogenized (51), and protein concentrations measured using the BCA protein assay (Thermo Scientific). Proteins were separated by SDS-PAGE (4\%-15\%) (Bio-Rad) and transferred to nitrocellulose (Bio-Rad). After blocking, membranes were incubated in rabbit antiPDE6b Ab (1:1,000; catalog PA1-722; Thermo Scientific) overnight at $4^{\circ} \mathrm{C}$, washed, and then incubated in goat anti-rabbit IgG-HRP secondary Ab (1:2,000; catalog sc-2004; Santa Cruz Biotechnology Inc.) for 1 hour at room temperature. $\beta$-actin loading control was detected using mouse anti- $\beta$-actin $\mathrm{Ab}$ (1:1,000; catalog ab125248; Abcam). 
Membranes were visualized by chemiluminescence detection (EMD Millipore) using Biomax film (Kodak).

ERG. Mice were dark adapted overnight, and recordings were carried out under dim red light illumination. Mice were first anesthetized by i.p. injection of $0.1 \mathrm{ml} / 10 \mathrm{~g} \mathrm{BW}$ of anesthetic solution ( $1 \mathrm{ml}$ of 100 $\mathrm{mg} / \mathrm{ml}$ ketamine and $0.1 \mathrm{ml}$ of $20 \mathrm{mg} / \mathrm{ml}$ xylazine in $8.9 \mathrm{ml} \mathrm{PBS}$ ). Body temperature was maintained at $37^{\circ} \mathrm{C}$ using a heating pad. Mouse pupils were dilated by topical administration of $2.5 \%$ phenylephrine hydrochloride and $1 \%$ tropicamide. Handmade electrodes were placed on the corneas, and gonioscopic prism solution (Alcon) was applied to each eye. ERG responses were recorded simultaneously from both eyes using an Espion Visual Electrophysiological System (Diagnosys). Rod and mixed rod-cone responses were recorded in dark-adapted mice using pulses of 0.001 and $3 \mathrm{~cd} \times \mathrm{s} / \mathrm{m}^{2}$ (white $6500 \mathrm{~K}$ ), respectively. Then, mice were light adapted for photopic responses for at least 10 minutes in the Ganzfeld dome. Recordings were carried out under rod-suppressing continuous background illumination of $30 \mathrm{~cd} / \mathrm{m}^{2}$ (white $6500 \mathrm{~K}$ ), and cone responses were recorded using pulses of $30 \mathrm{~cd} \times \mathrm{s} / \mathrm{m}^{2}$ (xenon).

Statistics. All data were analyzed using Stata 12 and R 3.1.1. Twotailed $t$ tests were used to analyze the data, and $P$ values of less than 0.05 were considered significant. To compare ERG data between groups at a single time point, we first averaged the ERG amplitude data for the 2 eyes of each mouse and then averaged the data for all of the mice within a single group (e.g., untreated mutants). To compare the mean ERG amplitudes between groups, a linear regression model was fit (where the outcome was the measurement and the predictors in the model were the group indicators), and pairwise comparisons between the groups were performed.

To compare ERG data between 2 time points (e.g., between weeks 10 and 32) for a single group, we carried out 2 distinct statistical analyses - one for mutants and a second for treated mutants. This is because while treated mutant mice were subjected to repeated ERG recordings, untreated mutant mice were subjected to a single ERG recording, at 1 time point. For treated mutant mice, we calculated the change over time for each eye and then calculated the average change per mouse; we then calculated the average for all mice within this group. In this way, we were able to study the within-subject changes for treated mutant mice. In untreated mutants, we first calculated the average ERG amplitude for the 2 eyes of each mouse, at the single time point, and then calculated the average for all mice within the mutant group. To compare mean outcomes between time points, we performed a 2-sample $t$ test.

For analyses of OS lengths and ONL thickness, multiple sections were taken from a retina at 1 time point. For each measurement (e.g., rod OS length), values from multiple sections were averaged. Then, a linear regression model was fit, where the outcome was a structural measurement and the predictors were the group indicators, and pairwise comparisons between groups were performed.

Study approval. All experiments were approved by the IACUC of Columbia University. Mice were used in accordance with the Statement for the Use of Animals in Ophthalmic and Vision Research of the Association for Research in Vision and Ophthalmology and the Policy for the Use of Animals in Neuroscience Research of the Society for Neuroscience.

\section{Acknowledgments}

We thank Richard Davis and members of the Jonas Stem Cell Laboratory and the Brown Glaucoma Laboratory for sharing ideas and equipment and, especially, Lawrence Chan, Winston Lee, and Rebecca Tuttle for critical reviews of the manuscript. We also thank Sara Lopez-Pintado for comments on the statistical analyses. The Columbia Confocal Facility and the animal facilities are supported by NIH Core grant 5P30EY019007, NCI Core grant 5P30CA013696, and unrestricted funds from Research to Prevent Blindness (RPB) and Columbia University. S.H. Tsang is a member of the RD-CURE Consortium and is supported by the Tistou and Charlotte Kerstan Foundation; the Foundation for Research on Degenerative Ophthalmic Conditions (FRODO); NIH grants R01EY018213, R21AG050437, and R01EY024698; the RPB Physician-Scientist Award; the Schneeweiss Stem Cell Fund; New York State (N09G-302 and N13G-275); the Foundation Fighting Blindness New York Regional Research Center Grant (C-NY050705-0312); Kobi and Nancy Karp; the Crowley Research Fund; the Professor Gertrude Rothschild Stem Cell Foundation; the Gebroe Family Foundation; and the Burroughs Wellcome Career Awards in Biomedical Sciences Program.

Address correspondence to: Stephen H. Tsang, $635 \mathrm{~W} .165^{\text {th }}$ Street, $5^{\text {th }}$ Floor New York, New York 10032, USA. Phone: 212.342.1189; E-mail:sht2@columbia.edu.
1. Hartong DT, Berson EL, Dryja TP. Retinitis pigmentosa. Lancet. 2006;368(9549):1795-1809.

2. Berson EL. Retinitis pigmentosa. The Friedenwald Lecture. Invest Ophthalmol Vis Sci. 1993;34(5):1659-1676.

3. Davis RJ, et al. Therapeutic margins in a novel preclinical model of retinitis pigmentosa. J Neurosci. 2013;33(33):13475-13483.

4. Sahel JA, Roska B. Gene therapy for blindness. Annu Rev Neurosci. 2013;36:467-488.

5. Petrs-Silva H, Linden R. Advances in gene therapy technologies to treat retinitis pigmentosa. Clin Ophthalmol. 2014;8:127-136.

6. Thompson DA, et al. Advancing therapeutic strategies for inherited retinal degeneration: recommendations from the Monaciano Symposium. Invest Ophthalmol Vis Sci. 2015;56(2):918-931.

7. Mitamura Y, Mitamura-Aizawa S, Nagasawa T,
Katome T, Eguchi H, Naito T. Diagnostic imaging in patients with retinitis pigmentosa. J Med Invest. 2012;59(1-2):1-11.

8. Cepko CL, Vandenberghe LH. Retinal gene therapy coming of age. Hum Gene Ther. 2013;24(3):242-244.

9. McLaughlin ME, Ehrhart TL, Berson EL, Dryja TP. Mutation spectrum of the gene encoding the beta subunit of rod phosphodiesterase among patients with autosomal recessive retinitis pigmentosa. Proc Natl Acad Sci US A. 1995;92(8):3249-3253.

10. Avila-Fernandez A, et al. Mutation analysis of 272 Spanish families affected by autosomal recessive retinitis pigmentosa using a genotyping microarray. Mol Vis. 2010;16:2550-2558.

11. Huang SH, Pittler SJ, Huang X, Oliveira L, Berson EL, Dryja TP. Autosomal recessive retinitis pig- mentosa caused by mutations in the alpha subunit of rod cGMP phosphodiesterase. Nat Genet. 1995;11(4):468-471.

12. Hart AW, et al. Genotype-phenotype correlation of mouse pde6b mutations. Invest Ophthalmol Vis Sci. 2005;46(9):3443-3450.

13. Davis RJ, et al. Functional rescue of degenerating photoreceptors in mice homozygous for a hypomorphic cGMP phosphodiesterase $6 \mathrm{~b}$ allele (Pde6bH620Q). Invest Ophthalmol Vis Sci. 2008;49(11):5067-5076.

14. Chang B, Hawes NL, Hurd RE, Davisson MT, Nusinowitz S, Heckenlively JR. Retinal degeneration mutants in the mouse. Vision Res. 2002;42(4):517-525.

15. Soriano P. Generalized lacZ expression with the ROSA26 Cre reporter strain. Nat Genet. 1999;21(1):70-71. 
16. Furukawa A, Koike C, Lippincott P, Cepko CL, Furukawa T. The mouse Crx 5'-upstream transgene sequence directs cell-specific and developmentally regulated expression in retinal photoreceptor cells. J Neurosci. 2002;22(5):1640-1647.

17. Young RW. Cell death during differentiation of the retina in the mouse. JComp Neurol. 1984;229(3):362-373.

18. Swaroop A, Kim D, Forrest D. Transcriptional regulation of photoreceptor development and homeostasis in the mammalian retina. Nat Rev Neurosci. 2010;11(8):563-576.

19. Wert KJ, Lin JH, Tsang SH. General pathophysiology in retinal degeneration. Dev Ophthalmol. 2014;53:33-43.

20. Dejneka NS, et al. In utero gene therapy rescues vision in a murine model of congenital blindness. Mol Ther. 2004;9(2):182-188.

21. Bennicelli J, et al. Reversal of blindness in animal models of leber congenital amaurosis using optimized AAV2-mediated gene transfer. Mol Ther. 2008;16(3):458-465.

22. Clarke G, et al. A one-hit model of cell death in inherited neuronal degenerations. Nature. 2000;406(6792):195-199.

23. Bainbridge JW, et al. Effect of gene therapy on visual function in Leber's congenital amaurosis. N Engl JMed. 2008;358(21):2231-2239.

24. Cideciyan AV, et al. Human RPE65 gene therapy for Leber congenital amaurosis: persistence of early visual improvements and safety at 1 year. Hum Gene Ther. 2009;20(9):999-1004.

25. Maguire AM, et al. Age-dependent effects of RPE65 gene therapy for Leber's congenital amaurosis: a phase 1 dose-escalation trial. Lancet. 2009;374(9701):1597-1605.

26. Simonelli F, et al. Gene therapy for Leber's congenital amaurosis is safe and effective through 1.5 years after vector administration. Mol Ther. 2010;18(3):643-650.

27. Jacobson SG, et al. Gene therapy for leber congenital amaurosis caused by RPE65 mutations: safety and efficacy in 15 children and adults followed up to 3 years. Arch Ophthalmol.
2012;130(1):9-24.

28. Bennett J, et al. AAV2 gene therapy readministration in three adults with congenital blindness. $\mathrm{Sci}$ Transl Med. 2012;4(120):120ra115.

29. Ashtari M, et al. The human visual cortex responds to gene therapy-mediated recovery of retinal function. J Clin Invest. 2011;121(6):2160-2168.

30. Testa F, et al. Three-year follow-up after unilateral subretinal delivery of adeno-associated virus in patients with Leber congenital Amaurosis type 2. Ophthalmology. 2013;120(6):1283-1291.

31. Cideciyan AV, et al. Human retinal gene therapy for Leber congenital amaurosis shows advancing retinal degeneration despite enduring visual improvement. Proc Natl Acad Sci US A. 2013;110(6):E517-E525.

32. Bainbridge JW, et al. Long-term effect of gene therapy on Leber's congenital amaurosis. N Engl J Med. 2015;372(20):1887-1897.

33. Jacobson SG, et al. Improvement and decline in vision with gene therapy in childhood blindness. N Engl J Med. 2015;372(20):1920-1926.

34. Wright AF. Long-term effects of retinal gene therapy in childhood blindness. $N$ Engl J Med. 2015;372(20):1954-1955.

35. Sarra GM, et al. Gene replacement therapy in the retinal degeneration slow (rds) mouse: the effect on retinal degeneration following partial transduction of the retina. Hum Mol Genet. 2001;10(21):2353-2361.

36. Koch S, et al. Gene therapy restores vision and delays degeneration in the CNGB1(-/-) mouse model of retinitis pigmentosa. Hum Mol Genet. 2012;21(20):4486-4496.

37. Wert KJ, Davis RJ, Sancho-Pelluz J, Nishina PM, Tsang SH. Gene therapy provides long-term visual function in a pre-clinical model of retinitis pigmentosa. Hum Mol Genet. 2013;22(3):558-567.

38. Mowat FM, et al. RPE65 gene therapy slows cone loss in Rpe65-deficient dogs. Gene Ther. 2013;20(5):545-555.

39. Sahel JA. Saving cone cells in hereditary rod diseases: a possible role for rod-derived cone viability factor (RdCVF) therapy. Retina.
2005;25(8 suppl):S38-S39.

40. Peng B, Xiao J, Wang K, So KF, Tipoe GL, Lin B. Suppression of microglial activation is neuroprotective in a mouse model of human retinitis pigmentosa. J Neurosci. 2014;34(24):8139-8150.

41. Shen S, Sujirakul T, Tsang SH. Next-generation sequencing revealed a novel mutation in the gene encoding the beta subunit of rod phosphodiesterase. Ophthalmic Genet. 2014;35(3):142-150.

42. Vandenberghe LH, Auricchio A. Novel adenoassociated viral vectors for retinal gene therapy. Gene Ther. 2012;19(2):162-168.

43. Khani SC, et al. AAV-mediated expression targeting of rod and cone photoreceptors with a human rhodopsin kinase promoter. Invest Ophthalmol Vis Sci. 2007;48(9):3954-3961.

44. Dalkara D, et al. In vivo-directed evolution of a new adeno-associated virus for therapeutic outer retinal gene delivery from the vitreous. Sci Transl Med. 2013;5(189):189ra176.

45. Lin TC, Hsu CC, Chien KH, Hung KH, Peng CH, Chen SJ. Retinal stem cells and potential cell transplantation treatments. J Chin Med Assoc. 2014;77(11):556-561.

46. Busskamp V, et al. Genetic reactivation of cone photoreceptors restores visual responses in retinitis pigmentosa. Science. 2010;329(5990):413-417.

47. Jacobson SG, Sumaroka A, Luo X, Cideciyan AV. Retinal optogenetic therapies: clinical criteria for candidacy. Clin Genet. 2013;84(2):175-182.

48. Weiland JD, Humayun MS. Retinal prosthesis. IEEE Trans Biomed Eng. 2014;61(5):1412-1424.

49. Sancho-Pelluz J, et al. Mice with a D190N mutation in the gene encoding rhodopsin: a model for human autosomal-dominant retinitis pigmentosa. Mol Med. 2012;18:549-555.

50. Tsang SH, et al. Retinal degeneration in mice lacking the gamma subunit of the rod cGMP phosphodiesterase. Science. 1996;272(5264):1026-1029.

51. Tsang SH, et al. Role for the target enzyme in deactivation of photoreceptor $G$ protein in vivo. Science. 1998;282(5386):117-121. 